Interfaces and Free Boundaries 17 (2015), 245-262

DOI $10.4171 / \mathrm{IFB} / 341$

\title{
The integral of the normal and fluxes over sets of finite perimeter
}

\author{
IDO BRIGHT \\ Department of Applied Mathematics, University of Washington, Seattle, WA 98195, USA \\ E-mail:ibright@uw.edu \\ MONICA TORRES \\ Department of Mathematics, Purdue University, 150 N. University Street, West Layayette, IN \\ 47907-2067, USA \\ E-mail: torres@math.purdue.edu
}

[Received 19 June 2014 and in revised form 12 December 2014]

\begin{abstract}
Given two intersecting sets of finite perimeter, $E_{1}$ and $E_{2}$, with unit normals $\boldsymbol{v}_{1}$ and $\boldsymbol{v}_{2}$ respectively, we obtain a bound on the integral of $\boldsymbol{v}_{1}$ over the reduced boundary of $E_{1}$ inside $E_{2}$. This bound depends only on the perimeter of $E_{2}$. For any vector field $\boldsymbol{F}: \mathbb{R}^{n} \rightarrow \mathbb{R}^{n}$ with the property that $\boldsymbol{F} \in L^{\infty}$ and $\operatorname{div} \boldsymbol{F}$ is a (signed) Radon measure, we obtain bounds on the flux of $\boldsymbol{F}$ over the portion of the reduced boundary of $E_{1}$ inside $E_{2}$. These results are then applied to study the limit of surfaces with perimeter growing to infinity.
\end{abstract}

2010 Mathematics Subject Classification: Primary 28A75; Secondary 28A50.

Keywords: Gauss-Green theorem; divergence-measure fields; sets of finite perimeter; normal traces; shape optimization; occupational measures.

\section{Introduction}

An application of the Gauss-Green Theorem shows that the integral over the reduced boundary of any set of finite perimeter $E \subset \mathbb{R}^{n}$ of the normal vector field is the zero vector (see Lemma 3.3); namely,

$$
\int_{\partial^{*} E} \boldsymbol{v}(x) d \mathcal{H}^{n-1}(x)=\mathbf{0} .
$$

With the aid of (1.1) we prove in this paper an estimate of the integral of the normal over a surface that lies on the boundary of a set of finite perimeter (see Theorem 3.2). More precisely, given two intersecting sets of finite perimeter $E_{1}$ and $E_{2}$ with unit normals $\boldsymbol{v}_{1}$ and $\boldsymbol{v}_{2}$ respectively, we show in Theorem 3.2 that

$$
\left|\int_{\partial^{*} E_{1} \cap E_{2}^{1}} \boldsymbol{v}_{1}(x) d \mathcal{H}^{n-1}\right| \leqslant \frac{1}{2} \mathcal{H}^{n-1}\left(\partial^{*} E_{2}\right),
$$

where $\partial^{*} E_{1}$ is the reduced boundary of $E_{1}$ and $E_{2}^{1}$ is the measure-theoretic interior of $E_{2}$. The significance of this result is that the bound depends only on the perimeter of $E_{2}$. We then use estimate (1.2) to study the limit of sets with perimeter growing to infinity.

Given a vector field $\boldsymbol{F}$, we ask the question whether we can estimate the integral of $\boldsymbol{F} \cdot \boldsymbol{v}$ over a surface $S$ that lies on the boundary of a set of finite perimeter $E$. That is, we would like to estimate the flux of the vector field $\boldsymbol{F}$ across $S$. This is a delicate question and the answer depends on the regularity of $\boldsymbol{F}$. Indeed, if the vector field $\boldsymbol{F}$ is continuous then $\boldsymbol{F} \cdot \boldsymbol{v}$ is defined on any surface 
$S \subset \mathbb{R}^{n}$. However, a vector field $\boldsymbol{F} \in L^{p}\left(\mathbb{R}^{n} ; \mathbb{R}^{n}\right)$ is defined only up to a set of Lebesgue measure zero and therefore, given any surface $S \subset \mathbb{R}^{n}$, such $\boldsymbol{F}$ might not even be defined on $S$. However, it has been proved in Chen-Torres-Ziemer [8,9] and Šilhavý [17] that if $\boldsymbol{F} \in L^{\infty}\left(\mathbb{R}^{n} ; \mathbb{R}^{n}\right)$ satisfies that $\operatorname{div} \boldsymbol{F}$ is a Radon measure (we will refer to these vector fields as divergence-measure fields), then $\boldsymbol{F}$ has an interior normal trace on the reduced boundary of any set of finite perimeter $E$. This normal trace is a function, denoted as $\mathscr{F} \cdot v$, that satisfies $\mathscr{F} \cdot v \in L^{\infty}\left(\partial^{*} E\right)$. Therefore, the flux of a divergence-measure vector field $\boldsymbol{F} \in L^{\infty}$ through $S$,

$$
\int_{S} \mathscr{F} \cdot v(x) d \mathcal{H}^{n-1}(x)
$$

is defined on any surface $S$ that is the boundary of a set of finite perimeter. In Theorems 4.2 and 4.3 we obtain estimates on the flux of bounded divergence-measure vector fields using the Gauss-Green formula proved in [8, 9, 17] (see also Frid [14] and the references therein). We refer to the book of Dafermos [10, Chapter 1] and [8] for the connections between fluxes of divergence-measure fields and the theory of hyperbolic conservation laws.

We then apply our main Theorem 3.2 to the analysis of sets with perimeter growing to infinity (see Theorem 5.2). Our results rely on the analysis of occupational measures, which are probability measures essentially supported on the boundaries of sets of finite perimeter. Occupational measures appear in the study of stochastic processes, and also in the context of optimization in the study of infinite horizon optimal control (see Finlay-Gaitsgory-Lebedev [13], Artstein-Bright [2] and Gaitsgory-Quincampoix [15]).

The aforementioned bounds extend previous bounds by Bright-Lee [3] from the smooth to non-smooth settings. Some of the results on sets of finite perimeter presented in this paper, and in particular Theorem 5.2, will be used by the authors to study shape optimization problems in a forthcoming paper.

The organization of this paper is as follows. In Section 2 we present some facts about sets of finite perimeter and we state the Gauss-Green formula that we will use in the paper, as well as other results. In Section 3 we obtain our estimate on the integral of the normal. In Section 4 we show that the same technique used in Section 3 can be applied to estimate the flux of bounded divergencemeasure fields. Finally, in Section 5 we introduce occupational measures and use them to study the limit of sets with perimeter growing to infinity.

\section{Sets of finite perimeter and the Gauss-Green formula for divergence-measure fields}

In this section we first recall some properties of Radon measures, sets of finite perimeter, and related $B V$ functions ( $[1,11])$. For the sake of completeness, we start with some basic notions and definitions. First, denote by $\mathcal{H}^{n-1}$ the $(n-1)$-dimensional Hausdorff measure in $\mathbb{R}^{n}$, and by $\mathcal{L}^{n}$ the Lebesgue measure in $\mathbb{R}^{n}$ (recall that $\mathcal{L}^{n}=\mathcal{H}^{n}$ ). The space $\mathfrak{M}(\Omega)$ consists of all finite Radon measures $\mu$ in the open set $\Omega$; that is, the total variation of $\mu$, denoted as $\|\mu\|$, satisfies $\|\mu\|(\Omega)<\infty$. For any set $E \subset \mathbb{R}^{n}$, we denote by $\bar{E}$ and $\partial E$ its topological closure and boundary, respectively. We denote by $E \Subset \mathbb{R}^{n}$ that the closure of $E$ is compact and contained in $\mathbb{R}^{n}$. The complement of the set $E$ is denoted by $E^{c}=\mathbb{R}^{n} \backslash E$. Also, we denote $B(x, r)$ as the open ball of radius $r$ and center at $x$. The symmetric difference of sets is denoted by

$$
A \Delta B:=(A \backslash B) \cup(B \backslash A)
$$


Definition 2.1. For every $\alpha \in[0,1]$ and every $\mathcal{L}^{n}$-measurable set $E \subset \mathbb{R}^{n}$, define

$$
E^{\alpha}:=\left\{y \in \mathbb{R}^{n}: D(E, y)=\alpha\right\},
$$

where

$$
D(E, y):=\lim _{r \rightarrow 0} \frac{\mathcal{L}^{n}(E \cap B(y, r))}{\mathcal{L}^{n}(B(y, r))} .
$$

Then $E^{\alpha}$ is the set of all points with density $\alpha$. We define the measure-theoretic boundary of $E$, $\partial^{m} E$, as

$$
\partial^{m} E:=\mathbb{R}^{n} \backslash\left(E^{0} \cup E^{1}\right) .
$$

Definition 2.2. Let $E \subset \mathbb{R}^{n}$. We say that $E$ is a set of finite perimeter in $\Omega$ if

$$
\operatorname{Per}(E, \Omega):=\sup \left\{\int_{E} \operatorname{div} \varphi d x: \varphi \in C_{c}^{1}(\Omega),\|\varphi\|_{\infty} \leqslant 1\right\}<\infty .
$$

We say that $E$ is a set of locally finite perimeter if it is a set of finite perimeter in every $\Omega \Subset \mathbb{R}^{n}$.

Definition 2.3. A function $f \in L^{1}(\Omega)$ is called a function of bounded variation if its distributional derivative $D f$ is a finite Radon vector measure; that is, $\|D f\|(\Omega)<\infty$, where $\|D f\|$ is the total variation of $D f$. Notationally, we write $f \in B V(\Omega)$.

Remark 2.4. If $E$ is a set of finite perimeter in $\mathbb{R}^{n}$ and $E$ has finite Lebesgue measure in $\mathbb{R}^{n}$, namely, $\mathcal{L}^{n}(E)<\infty$ then its characteristic function, denoted as $\chi_{E}$, is in $B V\left(\mathbb{R}^{n}\right)$. Moreover,

$$
\left\|D \chi_{E}\right\|=\mathcal{H}^{n-1}\left\llcorner\partial^{*} E,\right.
$$

where $\partial^{*} E$ is the reduced boundary of the set $E$ (see the definition below).

Definition 2.5. Let $E$ be a set of finite perimeter in $\mathbb{R}^{n}$. The reduced boundary of $E$, denoted as $\partial^{*} E$, is the set of all points $y \in \mathbb{R}^{n}$ such that

1. $\left\|D \chi_{E}\right\|(B(y, r))>0$ for all $r>0$;

2. The limit $\boldsymbol{v}_{E}(y):=\lim _{r \rightarrow 0} \frac{D \chi_{E}(B(y, r))}{\left\|D \chi_{E}\right\|(B(y, r))}$ exists and $\left|\boldsymbol{v}_{E}(y)\right|=1$.

Remark 2.6. Throughout the paper we use indistinctly the notation

$$
\operatorname{Per}(E)=\operatorname{Per}\left(E, \mathbb{R}^{n}\right)=\mathcal{H}^{n-1}\left(\partial^{*} E\right)
$$

to denote the perimeter of the set $E$.

The unit vector, $\boldsymbol{v}_{E}(y)$, is called the measure-theoretic interior unit normal to $E$ at $y$ (we sometimes write $\boldsymbol{v}$ instead of $\boldsymbol{v}_{E}$ for notational simplicity). In view of the following, we see that $\boldsymbol{v}$ is aptly named because $\boldsymbol{v}(y)$ satisfies

$$
D(\{x:(x-y) \cdot \boldsymbol{v}(y)>0, x \notin E\} \cup\{x:(x-y) \cdot v(y)<0, x \in E\}, y)=0,
$$

which means that $\boldsymbol{v}_{E}(y)$ is the interior unit normal to $E$ in the sense that $E$ (in the limit and in measure) lies in the appropriate half-space determined by the hyperplane orthogonal to $\boldsymbol{v}$.

The following result is due to Federer (see also [18] Lemma 5.9.5. and [1], Theorem 3.61): 
THEOREM 2.7 If $E$ is a set of finite perimeter in $\mathbb{R}^{n}$, then

$$
\partial^{*} E \subset E^{\frac{1}{2}} \subset \partial^{m} E, \quad \mathcal{H}^{n-1}\left(\mathbb{R}^{n} \backslash\left(E^{0} \cup \partial^{*} E \cup E^{1}\right)\right)=0 .
$$

In particular, $E$ has density either 0 or $1 / 2$ or 1 at $\mathcal{H}^{n-1}$-a.e. $x \in \mathbb{R}^{n}$ and $\mathcal{H}^{n-1}$-a.e. $x \in \partial^{m} E$ belongs to $\partial^{*} E$.

We will refer to the sets $E^{0}$ and $E^{1}$ as the measure-theoretic exterior and interior of $E$. We note that, in general, the sets $E^{0}$ and $E^{1}$ do not coincide with the topological exterior and interior of the set $E$. The sets $E^{0}$ and $E^{1}$ also motivate the definition of measure-theoretic boundary. We note that (2.5) implies, for any set $E \Subset \mathbb{R}^{n}$ of finite perimeter,

$$
\mathbb{R}^{n}=E^{1} \cup \partial^{*} E \cup E^{0} \cup n
$$

where $\mathcal{H}^{n-1}(\eta)=0$.

We recall that the $B V$ space, the space of functions of bounded variation, in fact represents equivalence classes of functions so that, when a function in a class is changed on a set of $\mathcal{L}^{n}$ measure zero, it remains in this class. The same is true for sets of finite perimeter because, by definition, the characteristic function $\chi_{E}$ of a set of finite perimeter, $E$, is a function of bounded variation. Thus, it follows that $E$ may be altered by a set of $\mathcal{L}^{n}$-measure zero and still determine the same essential boundary $\partial^{m} E$.

Remark 2.8. Throughout this paper, we will choose a preferred representative for $E$ and thereby adopt the following convention:

$$
E=E^{1} \cup \partial^{m} E
$$

The following result by Šilhavý [16, Proposition 3.8.5, page 81], which is easily verified, will be needed in the sequel.

PROPOSITION 2.9 If $E_{1}, E_{2}$ are sets of finite perimeter in $\mathbb{R}^{n}$, with corresponding measure theoretic normal vectors $\boldsymbol{v}_{1}$ and $\boldsymbol{v}_{2}$, then:

1. Up to a set of zero $\mathfrak{H}^{n-1}$-measure

$$
\partial^{m}\left(E_{1} \cap E_{2}\right)=\left(\partial^{m} E_{1} \cap E_{2}^{1}\right) \cup\left(E_{1}^{1} \cap \partial^{m} E_{2}\right) \cup \delta,
$$

where $\delta=\left\{x \in \partial^{*} E_{1} \cap \partial^{*} E_{2} \mid \boldsymbol{v}_{1}(x)=\boldsymbol{v}_{2}(x)\right\}$.

2. Let $E_{3}=E_{1} \cap E_{2}$ then its corresponding measure theoretic normal vector $\boldsymbol{v}_{3}$ satisfies, for $\mathcal{H}^{n-1}$-a.e point $x \in \partial^{*} E_{3}$,

$$
\boldsymbol{v}_{3}(x)= \begin{cases}\boldsymbol{v}_{1}(x) & x \in \partial^{*} E_{1} \cap E_{2}^{1} \\ \boldsymbol{v}_{2}(x) & x \in \partial^{*} E_{2} \cap E_{1}^{1} \\ \boldsymbol{v}_{1}(x)=\boldsymbol{v}_{2}(x) & x \in S\end{cases}
$$

We will use the following

LEMMA 2.10 If $E \subset \mathbb{R}^{n}$ is a set of finite perimeter then, for every $x \in \mathbb{R}^{n}$ and almost every $r>0$, $\mathcal{H}^{n-1}\left(\partial^{*} E \cap \partial B(x, r)\right)=0$. 
Proof. By the coarea formula we have

$$
0=\mathcal{L}^{n}\left(\partial^{*} E\right)=\int_{0}^{\infty} \mathcal{H}^{n-1}\left(\partial B(x, r) \cap \partial^{*} E\right) d \mathcal{L}(r)
$$

which clearly implies the desired result.

Definition 2.11. The vector field $\boldsymbol{F} \in L^{\infty}\left(\Omega ; \mathbb{R}^{n}\right)$ is called a divergence measure field in $\Omega$ if div $\boldsymbol{F}$, the distributional divergence of $\boldsymbol{F}$, is a finite Radon measure in $\Omega$. That is $\|\operatorname{div} \boldsymbol{F}\|(\Omega)<\infty$.

In this paper we use the Gauss-Green Formula (see (2.9) below) for bounded divergencemeasure fields $\boldsymbol{F}$ over sets of finite perimeter $E$, which was proved in Šilhavý [17], Chen-Torres [8] and Chen-Torres-Ziemer [9]. In [8], the interior normal trace $\mathscr{F} \cdot v \in L^{\infty}\left(\partial^{*} E\right)$ was obtained using a product rule for divergence-measure fields. In this paper we prefer to use Šilhavý's formula for the trace since it is expressed in terms of the normal vector $v(x)$ (see 2.7 below). In Chen-TorresZiemer [9], the normal trace $\mathscr{F} \cdot \boldsymbol{v}$ was constructed with a different approach; namely, $\mathscr{F} \cdot \boldsymbol{v}$ was obtained as the limit of the classical normal traces $\boldsymbol{F} \cdot \boldsymbol{v}$, which are defined on almost every surface that approximate the reduced boundary of $E$. This requires to show that a set of finite perimeter $E$ can be approximated with smooth surfaces from "one-side" of the reduced boundary with respect to the measure $\operatorname{div} \boldsymbol{F}$.

TheOREM 2.12 Suppose $\boldsymbol{F}: \mathbb{R}^{n} \rightarrow \mathbb{R}^{n}$ is a vector field with the properties $\boldsymbol{F} \in L^{\infty}\left(\mathbb{R}^{n} ; \mathbb{R}^{n}\right)$ and $\operatorname{div} \boldsymbol{F} \in \boldsymbol{m}\left(\mathbb{R}^{n}\right)$; that is, $\boldsymbol{F}$ is a divergence-measure field in $\mathbb{R}^{n}$. Let $E \subset \mathbb{R}^{n}$ be a set of finite perimeter. Then the interior normal trace of $\boldsymbol{F}$ on $\partial^{*} E$, denoted as $\mathscr{F} \cdot \boldsymbol{v}$, satisfies $\mathscr{F} \cdot \boldsymbol{v} \in L^{\infty}\left(\partial^{*} E\right)$ and is given, for $\mathcal{H}^{n-1}$-almost every $x \in \partial^{*} E$, by the formula $\mathscr{F} \cdot v(x)=T_{\boldsymbol{F}}(x, \boldsymbol{v}(x))$, where

$$
T_{\boldsymbol{F}}(x, \boldsymbol{v}(x))=\lim _{r \rightarrow 0} \frac{n}{\omega_{n-1} r^{n}} \int_{B(x, r, \boldsymbol{v}(x))} \boldsymbol{F}(y) \cdot \frac{y-x}{|y-x|} d \mathcal{L}^{n}(y),
$$

where $B(x, r, v(x))=B(x, r) \cap\{y \mid(y-x) \cdot v(x)>0\}$ and $\omega_{n-1}$ is the volume of the $(n-1)$ dimensional unit ball in $\mathbb{R}^{n-1}$. For every Lipschitz continuous test function with compact support $\phi$

$$
-\int_{\partial^{m} E} \phi(x) \mathscr{F} \cdot \boldsymbol{v}(x) d \mathcal{H}^{n-1}(x)=\int_{E^{1}} \phi(x) \operatorname{div} \boldsymbol{F}+\int_{E^{1}} \nabla \phi(x) \cdot \boldsymbol{F}(x) d \mathcal{L}^{n}(x) .
$$

In particular, if $E \Subset \mathbb{R}^{n}$ then

$$
-\int_{\partial^{m} E} \mathscr{F} \cdot \boldsymbol{v}(x) d \mathcal{H}^{n-1}(x)=\int_{E^{1}} \operatorname{div} \boldsymbol{F} .
$$

Moreover,

$$
\|\mathscr{F} \cdot \boldsymbol{v}\|_{\infty ; \partial^{*} E} \leqslant\|\boldsymbol{F}\|_{\infty ; \mathbb{R}^{n}}
$$

It was proven in Chen-Torres-Ziemer [9] (and it is also implied in Šilhavý [17]) that if $\boldsymbol{F}$ is continuous then $\mathscr{F} \cdot \boldsymbol{v}=\boldsymbol{F} \cdot \boldsymbol{v}$, the classical dot product, which is defined on any surface. Hence, we have: 
THEOREM 2.13 If $\boldsymbol{F}$ is continuous, the above Gauss-Green formula reduces to

$$
-\int_{\partial^{m} E} \phi(x) \boldsymbol{F}(x) \cdot \boldsymbol{v}(x) d \mathcal{H}^{n-1}(x)=\int_{E^{1}} \phi(x) \operatorname{div} \boldsymbol{F}+\int_{E^{1}} \nabla \phi(x) \cdot \boldsymbol{F}(x) d \mathcal{L}^{n}(x),
$$

for every Lipschitz continuous test function with compact support $\phi$. In particular, if $E \Subset \mathbb{R}^{n}$ we have

$$
-\int_{\partial^{m} E} \boldsymbol{F}(x) \cdot \boldsymbol{v}(x) d \mathcal{H}^{n-1}(x)=\int_{E^{1}} \operatorname{div} \boldsymbol{F} .
$$

\section{Estimates on the normal vector}

In this section we verify bounds on the integrals of the normal vector over the boundary of a set of finite perimeter. We recall that throughout the paper, whenever $E$ is a set of finite perimeter, we imply that $E$ is the precise representative $E:=E^{1} \cup \partial^{m} E$ (see Remark 2.8).

We first prove the following:

LEMMA 3.1 Let $E \subset \mathbb{R}^{n}$ be a set of finite perimeter and $O \subset \mathbb{R}^{n}$ an open set. Then

$$
E^{1} \cap O=(E \cap O)^{1} \text { and } E^{0} \cap O=(E \cap O)^{0} .
$$

Proof. Let $x \in E^{1} \cap O$. Since $x \in E^{1}$;

$$
\lim _{r \rightarrow 0} \frac{\mathcal{L}^{n}(E \cap B(x, r))}{\mathcal{L}^{n}(B(x, r))}=1 .
$$

Since $x$ is in the open set $O, B(x, r) \subset O$ for $r$ small enough. Thus:

$$
\begin{aligned}
\lim _{r \rightarrow 0} \frac{\mathcal{L}^{n}(E \cap B(x, r))}{\mathcal{L}^{n}(B(x, r))} & =\lim _{r \rightarrow 0} \frac{\mathcal{L}^{n}(E \cap B(x, r) \cap O)}{\mathcal{L}^{n}(B(x, r))} \\
& =\lim _{r \rightarrow 0} \frac{\mathcal{L}^{n}((E \cap O) \cap B(x, r))}{\mathcal{L}^{n}(B(x, r))} \\
& =1 .
\end{aligned}
$$

This implies that $x \in(E \cap O)^{1}$. Conversely, let $x \in(E \cap O)^{1}$. Thus,

$$
\lim _{r \rightarrow 0} \frac{\mathcal{L}^{n}((E \cap O) \cap B(x, r))}{\mathcal{L}^{n}(B(x, r))}=1
$$

that is,

$$
\lim _{r \rightarrow 0} \frac{\mathcal{L}^{n}(E \cap(O \cap B(x, r)))}{\mathcal{L}^{n}(B(x, r))}=1 .
$$

Notice that if a point $y$ satisfies $y \notin O$, then

$$
\lim _{r \rightarrow 0} \frac{\mathcal{L}^{n}(E \cap(O \cap B(y, r)))}{\mathcal{L}^{n}(B(y, r))} \leqslant \frac{1}{2},
$$


and this together with (3.1) implies that $x \in O$. Hence, since $O$ is open it follows that $B(x, r) \subset O$ for $r$ small enough. Thus,

$$
1=\lim _{r \rightarrow 0} \frac{\mathcal{L}^{n}(E \cap(O \cap B(x, r)))}{\mathcal{L}^{n}(B(x, r))}=\lim _{r \rightarrow 0} \frac{\mathcal{L}^{n}(E \cap B(x, r))}{\mathcal{L}^{n}(B(x, r))},
$$

and hence $x \in E^{1}$. We conclude that $x \in E^{1} \cap O$.

The second equality follows from the fact that

$$
x \in E^{0} \Longleftrightarrow x \in\left(E^{0} \cup \partial^{m} E\right)^{1} .
$$

We now state our main result:

THEOREM 3.2 Let $E_{1}, E_{2} \subset \mathbb{R}^{n}$ be sets of finite perimeter, then for $F=E_{2}, E_{2}^{1}, E_{2}^{0}$ or $E_{2}^{0} \cup \partial^{m} E_{2}$

$$
\left|\int_{\partial^{*} E_{1} \cap F} \boldsymbol{v}(x) d \mathcal{H}^{n-1}(x)\right| \leqslant \frac{\mathcal{H}^{n-1}\left(\partial^{*} E_{2}\right)}{2} .
$$

In order to prove the main result we use the following Lemma.

Lemma 3.3 Let $E \subset \mathbb{R}^{n}$ be a set of finite perimeter. Then,

$$
\int_{\partial^{*} E} \boldsymbol{v}(x) d \mathcal{H}^{n-1}(x)=\mathbf{0} .
$$

Proof. By replacing $E$ by $E^{0} \cup \partial^{m} E$, if necessary, we can assume that $\mathcal{L}^{n}(E)<\infty$. Let $\boldsymbol{F}_{i} \equiv$ $(0, \ldots, 1, \ldots, 0)$ (the $i$-th position is 1$)$ and note that $\boldsymbol{F}_{i}$ is a continuous vector field. We consider first the case $E \Subset \mathbb{R}^{n}$ and use the Gauss-Green formula (2.11) to obtain,

$$
\int_{E^{1}} \operatorname{div} \boldsymbol{F}_{i}(x) d \mathcal{L}^{n}(x)=0=-\int_{\partial^{*} E} v_{i}(y) d \mathcal{H}^{n-1}(y),
$$

where $\boldsymbol{v}(y)=\left(v_{1}(y), \ldots, v_{n}(y)\right)$. Hence,

$$
\int_{\partial^{*} E} v_{i}(x) d \mathcal{H}^{n-1}(x)=0, i=1,2, \ldots, n .
$$

We conclude

$$
\int_{\partial^{*} E} \boldsymbol{v}(x) d \mathcal{H}^{n-1}(x)=\mathbf{0} .
$$

Otherwise, when $E$ is not bounded, there exists $\epsilon>0$ and an open ball $B$ satisfying

$$
\mathcal{H}^{n-1}\left(\partial B \cap \partial^{*} E\right)=0, \mathcal{H}^{n-1}\left(\partial^{*} E \cap B^{c}\right)<\epsilon \text { and } \mathcal{H}^{n-1}(\partial B \cap E)<\epsilon .
$$

Indeed, using Lemma 2.10, for almost every $r, \mathcal{H}^{n-1}\left(\partial B(\mathbf{0}, r) \cap \partial^{*} E\right)=0$, and, clearly, $\mathcal{H}^{n-1}\left(\partial^{*} E \backslash B(\mathbf{0}, r)\right)<\epsilon$ for every $r$ large enough. Also, notice that the coarea formula yields:

$$
\int_{0}^{\infty} \mathcal{H}^{n-1}(\partial B(\mathbf{0}, r) \cap E) d \mathcal{L}(r)=\mathcal{L}^{n}(E)<\infty,
$$


and hence the set $S=\left\{r \mid \mathcal{H}^{n-1}(\partial B(\mathbf{0}, r) \cap E) \geqslant \epsilon\right\}$ has finite measure. Therefore, the complement of this set, $\left\{r \mid \mathcal{H}^{n-1}(\partial B(\mathbf{0}, r) \cap E)<\epsilon\right\}$, has infinite measure. Hence, there exists an $r$ such that $\mathcal{H}^{n-1}(\partial B(\mathbf{0}, r) \cap E)<\epsilon$.

For every $i$ we obtain, using the Gauss-Green formula (2.11) and Proposition 2.9 (1),

$$
\int_{(E \cap B)^{1}} \operatorname{div} \boldsymbol{F}_{i}(x) d \mathcal{L}^{n}(x)=\mathbf{0}=-\int_{\partial^{*} E \cap B} v_{i}(x) d \mathcal{H}^{n-1}(x)-\int_{\partial B \cap E^{1}} v_{i}(x) d \mathcal{H}^{n-1}(x) .
$$

This implies that

$$
\begin{aligned}
\left|\int_{\partial^{*} E} v_{i}(x) d \mathcal{H}^{n-1}(x)\right| & \leqslant\left|\int_{\partial^{*} E \cap B} v_{i}(x) d \mathcal{H}^{n-1}(x)\right|+\left|\int_{\partial^{*} E \backslash B} v_{i}(x) d \mathcal{H}^{n-1}(x)\right| \\
& \leqslant\left|\int_{\partial B \cap E^{1}} v_{i}(x) d \mathcal{H}^{n-1}(x)\right|+\epsilon, \text { from (3.4) and (3.5) } \\
& \leqslant 2 \epsilon, \quad \text { from (3.4). }
\end{aligned}
$$

Since $\epsilon$ is arbitrary the proof is complete.

COROLlary 3.4 Let $E \subset \mathbb{R}^{n}$ be a set of finite perimeter, $C \subset \partial^{*} E$. Then

$$
\left|\int_{C} \boldsymbol{v}(x) d \mathcal{H}^{n-1}(x)\right|=\left|\int_{\partial^{*} E \backslash C} \boldsymbol{v}(x) d \mathcal{H}^{n-1}(x)\right| \leqslant \frac{1}{2} \mathcal{H}^{n-1}\left(\partial^{*} E\right) .
$$

Proof. For any $C \subset \partial^{*} E$,

$$
\mathbf{0}=\int_{\partial^{*} E} \boldsymbol{v}(x) d \mathcal{H}^{n-1}(x)=\int_{C} \boldsymbol{v}(x) d \mathcal{H}^{n-1}(x)+\int_{\partial^{*} E \backslash C} v(x) d \mathcal{H}^{n-1}(x) .
$$

Therefore,

$$
\left|\int_{C} \boldsymbol{v}(x) d \mathcal{H}^{n-1}(x)\right|=\left|\int_{\partial^{*} E \backslash C} \boldsymbol{v}(x) d \mathcal{H}^{n-1}(x)\right|=\alpha(C) .
$$

Since

$$
\alpha(C) \leqslant \mathcal{H}^{n-1}(C) \text { and } \alpha(C) \leqslant \mathcal{H}^{n-1}\left(\partial^{*} E \backslash C\right)
$$

we obtain:

$$
2 \alpha(C) \leqslant \mathcal{H}^{n-1}(C)+\mathcal{H}^{n-1}\left(\partial^{*} E \backslash C\right)=\mathcal{H}^{n-1}\left(\partial^{*} E\right) .
$$

We conclude:

$$
\alpha(C) \leqslant \frac{1}{2} H^{n-1}\left(\partial^{*} E\right)
$$

Proof. [Proof of Theorem 3.2] Let $E_{3}=E_{1} \cap E_{2}$ and $\boldsymbol{v}_{1}, \boldsymbol{v}_{2}$ and $\boldsymbol{v}_{3}$ be the measure theoretic normal vectors corresponding to the sets $E_{1}, E_{2}$ and $E_{3}$, respectively. We first prove the theorem 
for $F=E_{2}^{1}$. By Lemma 3.3 and Proposition 2.9 we have that

$$
\begin{aligned}
\mathbf{0} & =\int_{\partial^{*}\left(E_{1} \cap E_{2}\right)} \boldsymbol{v}_{3}(x) d \mathcal{H}^{n-1}(x) \\
& =\int_{\partial^{*} E_{1} \cap E_{2}^{1}} \boldsymbol{v}_{1}(x) d \mathcal{H}^{n-1}(x)+\int_{\partial^{*} E_{2} \cap E_{1}^{1}} \boldsymbol{v}_{2}(x) d \mathcal{H}^{n-1}(x)+\int_{S} \boldsymbol{v}_{2}(x) d \mathcal{H}^{n-1}(x) \\
& =\int_{\partial^{*} E_{1} \cap E_{2}^{1}} \boldsymbol{v}_{1}(x) d \mathcal{H}^{n-1}(x)+\int_{C} \boldsymbol{v}_{2}(x) d \mathcal{H}^{n-1}(x),
\end{aligned}
$$

where $S=\left\{x \in \partial^{*} E_{1} \cap \partial^{*} E_{2} \mid \boldsymbol{v}_{1}(x)=\boldsymbol{v}_{2}(x)\right\}$, and $C$ is the disjoint union $S \cup\left(\partial^{*} E_{2} \cap E_{1}^{1}\right) \subset$ $\partial^{*} E_{2}$. From Corollary 3.4 we obtain

$$
\left|\int_{\partial^{*} E_{1} \cap E_{2}^{1}} \boldsymbol{v}_{1}(x) d \mathcal{H}^{n-1}(x)\right|=\left|\int_{C} \boldsymbol{v}_{2}(x) d \mathcal{H}^{n-1}(x)\right| \leqslant \frac{\mathcal{H}^{n-1}\left(\partial^{*} E_{2}\right)}{2},
$$

which proves the bound for $F=E_{2}^{1}$.

For $F=E_{2}^{0}$, the inequality follows by applying the latter result to $F=\left(E_{2}^{0} \cup \partial^{m} E_{2}\right)^{1}=E_{2}^{0}$. The proof for $F=E_{2}^{0} \cup \partial^{m} E_{2}$ follows from Lemma 3.3 since

$$
0=\int_{\partial^{*} E_{1}} \boldsymbol{v}_{1}(x) d \mathcal{H}^{n-1}(x)=\int_{\partial^{*} E_{1} \cap E_{2}^{1}} \boldsymbol{v}_{1}(x) d \mathcal{H}^{n-1}(x)+\int_{\partial^{*} E_{1} \cap\left(E_{2}^{0} \cup \partial^{m} E_{2}\right)} \boldsymbol{v}_{1}(x) d \mathcal{H}^{n-1}(x),
$$

and a similar argument holds for $F=E_{2}=E_{2}^{1} \cup \partial^{m} E_{2}$.

A weaker result follows when $E_{1}$ has only locally finite perimeter, and $E_{2}$ has finite measure. For this we need the following well known result.

LEMMA 3.5 Let $E \subset \mathbb{R}^{n}$ be a set of finite perimeter with finite measure and $K \subset \mathbb{R}^{n}$ a convex set. Then

$$
\operatorname{Per}(E \cap K) \leqslant \operatorname{Per}(E) \text {. }
$$

Proof. If $K=\mathbb{R}^{n}$ the result is clear. Otherwise, we first assume that $K=H=\left\{y \in \mathbb{R}^{n} \mid y \cdot v \leqslant r\right\}$ is the half-space defined by $v \in \mathbb{R}^{n}$ and $r \in \mathbb{R}$. Let $\tilde{E}=E \backslash H$. Proceeding as in Lemma 2.10 and using Proposition 2.9 (1) it follows that, up to a set of $\mathcal{H}^{n-1}$-measure zero and for almost every $r$,

$$
\partial^{*} \tilde{E}=\left(\partial^{*} E \cap H^{c}\right) \cup(E \cap \partial H)
$$

Therefore, by Lemma 3.3, for almost every $r$,

$$
\begin{aligned}
\mathbf{0} & =\int_{\partial^{*} \tilde{E}} \boldsymbol{v}(y) d \mathcal{H}^{n-1}(y)=\int_{\partial^{*} \tilde{E} \backslash H} \boldsymbol{v}(y) d \mathcal{H}^{n-1}(y)+\int_{\partial^{*} \tilde{E} \cap H} \boldsymbol{v}(y) d \mathcal{H}^{n-1}(y) \\
& =\int_{\partial^{*} E \backslash H} \boldsymbol{v}(y) d \mathcal{H}^{n-1}(y)+\int_{\partial H \cap E} \boldsymbol{v}(y) d \mathcal{H}^{n-1}(y) .
\end{aligned}
$$

(Note that since $E$ has finite measure then, by the coarea formula, the last term in (3.7) is finite for almost every $r$ ). 
Since $H$ is a half-space, then its normal vector is in the direction $\boldsymbol{v}_{\partial H} \equiv-v$, and by the triangle inequality the latter expression implies

$$
\mathcal{H}^{n-1}(\partial H \cap E)=\left|\int_{\partial^{*} E \backslash H} \boldsymbol{v}(y) d \mathcal{H}^{n-1}(y)\right| \leqslant \int_{\partial^{*} E \backslash H}|\boldsymbol{v}(y)| d \mathcal{H}^{n-1}(y)=\mathcal{H}^{n-1}\left(\partial^{*} E \backslash H\right) .
$$

From (3.8), we conclude that, for almost every $r$,

$$
\begin{aligned}
\operatorname{Per}(E) & =\mathcal{H}^{n-1}\left(\partial^{*} E\right)=\mathcal{H}^{n-1}\left(\partial^{*} E \cap H\right)+\mathcal{H}^{n-1}\left(\partial^{*} E \backslash H\right) \\
& \geqslant \mathcal{H}^{n-1}\left(\partial^{*} E \cap H\right)+\mathcal{H}^{n-1}(\partial H \cap E)=\operatorname{Per}(H \cap E) .
\end{aligned}
$$

For the general case, since $K$ is a convex set, the closure $\bar{K}$ is an intersection of countably many halfspaces. Thus, $\bar{K}$ is an intersection of countably many half-spaces satisfying (3.9). Writing $E \cap \bar{K}=$ $\lim _{N \rightarrow \infty} E \cap_{i=1}^{\mathrm{N}} H_{i}$ (in $L^{1}$ ), the lower semi-continuity of the perimeter implies

$$
\operatorname{Per}(E \cap \bar{K}) \leqslant \operatorname{Per}(E) \text {. }
$$

Moreover, since $K$ is convex we have that $K$ and $\bar{K}$ are Lebesgue equivalent and thus $\partial^{*} K=\partial^{*} \bar{K}$. Moreover, $\partial K=\partial \bar{K}$. Therefore, the reduced boundary and the topological boundary of $K$ are $\mathcal{H}^{n-1}$ equivalent, which implies that

$$
\operatorname{Per}(E \cap K)=\operatorname{Per}(E \cap \bar{K}) \leqslant \operatorname{Per}(E) \text {. }
$$

COROLlaRY 3.6 Let $E_{1} \subset \mathbb{R}^{n}$ be of locally finite perimeter and $E_{2} \subset \mathbb{R}^{n}$ of finite perimeter. If $E_{2}$ has finite measure then, for every open ball $B=B(x, r)$,

$$
\left|\int_{\partial^{*} E_{1} \cap E_{2}^{1} \cap B} v(x) d \mathcal{H}^{n-1}(x)\right| \leqslant \frac{\mathcal{H}^{n-1}\left(\partial^{*} E_{2}\right)}{2} .
$$

Furthermore, when $\mathcal{H}^{n-1}\left(\partial^{*} E_{1} \cap E_{2}\right)<\infty$, and, in particular, when $E_{2} \Subset \mathbb{R}^{n}$,

$$
\left|\int_{\partial^{*} E_{1} \cap E_{2}^{1}} \boldsymbol{v}(x) d \mathcal{H}^{n-1}(x)\right|,\left|\int_{\partial^{*} E_{1} \cap E_{2}} \boldsymbol{v}(x) d \mathcal{H}^{n-1}(x)\right| \leqslant \frac{\mathcal{H}^{n-1}\left(\partial^{*} E_{2}\right)}{2} .
$$

Proof. Let $\hat{B}$ be an open ball so that $\bar{B} \subset \hat{B}$. Consider the sets of finite perimeter $E:=\left(E_{1} \cap\right.$ $\hat{B})^{1} \cup \partial^{m}\left(E_{1} \cap \hat{B}\right)$ and $F=\left(E_{2} \cap B\right)^{1} \cup \partial^{m}\left(E_{2} \cap B\right)$. Thus, since $\partial \hat{B} \cap\left(E_{2}^{1} \cap B\right)=\emptyset$ and $B \subset \hat{B}$, Proposition 2.9 (1) yields

$$
\begin{aligned}
\left|\int_{\partial^{*} E_{1} \cap E_{2}^{1} \cap B} v(x) d \mathcal{H}^{n-1}(x)\right| & =\left|\int_{\partial^{*}\left(E_{1} \cap \hat{B}\right) \cap\left(E_{2}^{1} \cap B\right)} v(x) d \mathcal{H}^{n-1}(x)\right| \\
& =\left|\int_{\partial^{*}\left(E_{1} \cap \hat{B}\right) \cap\left(E_{2} \cap B\right)^{1}} v(x) d \mathcal{H}^{n-1}(x)\right|, \quad \text { by Lemma 3.1 }
\end{aligned}
$$




$$
\begin{aligned}
& =\left|\int_{\partial^{*} E \cap F^{1}} v(x) d \mathcal{H}^{n-1}(x)\right| \\
& \leqslant \frac{\mathcal{H}^{n-1}\left(\partial^{*} F\right)}{2}, \quad \text { by Theorem } 3.2 \\
& =\frac{\mathcal{H}^{n-1}\left(\partial^{*}\left(E_{2} \cap B\right)\right)}{2} \leqslant \frac{\mathcal{H}^{n-1}\left(\partial^{*} E_{2}\right)}{2}, \quad \text { by Lemma 3.5, }
\end{aligned}
$$

which proves inequality (3.11).

When $\mathcal{H}^{n-1}\left(\partial^{*} E_{1} \cap E_{2}\right)<\infty$, we fix $\epsilon>0$ and choose an open ball $B$, such that

$$
\mathcal{H}^{n-1}\left(\partial^{*} E_{1} \cap E_{2} \cap B^{c}\right)<\epsilon .
$$

By the previous inequality,

$$
\begin{aligned}
\left|\int_{\partial^{*} E_{1} \cap E_{2}^{1}} \boldsymbol{v}(x) d \mathcal{H}^{n-1}(x)\right| & =\left|\int_{\partial^{*} E_{1} \cap E_{2}^{1} \cap B} v(x) d \mathcal{H}^{n-1}(x)\right|+\left|\int_{\partial^{*} E_{1} \cap E_{2}^{1} \cap B^{c}} v(x) d \mathcal{H}^{n-1}(x)\right| \\
& \leqslant\left|\int_{\partial^{*} E_{1} \cap E_{2}^{1} \cap B} v(x) d \mathcal{H}^{n-1}(x)\right|+\epsilon \text {, by (3.13) } \\
& \leqslant \frac{\mathcal{H}^{n-1}\left(\partial^{*} E_{2}\right)}{2}+\epsilon .
\end{aligned}
$$

Taking $\epsilon$ to zero completes the proof of the first inequality in (3.12). For the second inequality, we proceed in the same way, integrating over $\partial^{*} E_{1} \cap E_{2}$ and then replacing $E_{2} \cap B$ by $F$. This introduces an extra term that can be controlled by (3.13). Finally, we apply Theorem 3.2 as before with $F$ in place of $E_{2}$.

\section{Estimates on the flux of a vector field.}

We recall that a vector field $\boldsymbol{F} \in L^{p}\left(\Omega ; \mathbb{R}^{n}\right)$ is defined up to a set of $\mathcal{L}^{n}$-measure zero. In particular, given $\boldsymbol{F} \in L^{\infty}\left(\Omega ; \mathbb{R}^{n}\right)$ and a surface $S \subset \mathbb{R}^{n}$, the vector field $\boldsymbol{F}$ might not be defined on $S$. However, it is proven in $[8,9,17]$ that if $\boldsymbol{F} \in L^{\infty}$ is a divergence-measure field and $S$ is a surface that is the boundary of a set of finite perimeter $E$, then $\boldsymbol{F}$ has an interior normal trace on $\partial^{*} E$, denoted as $\mathscr{F} \cdot \boldsymbol{v}$. Therefore, the flux of a divergence-measure vector field $\boldsymbol{F} \in L^{\infty}$,

$$
\int_{S} \mathscr{F} \cdot v(x) d \mathcal{H}^{n-1}(x)
$$

is defined on any surface $S$ that is the boundary of a set of finite perimeter. The same arguments used in Section 3 and the Gauss-Green formula (2.9) yield the following result that estimates the flux of a bounded divergence-measure vector field.

LEMma 4.1 Let $\boldsymbol{F} \in L^{\infty}\left(\mathbb{R}^{n} ; \mathbb{R}^{n}\right)$ be a divergence-measure vector field satisfying $\operatorname{div} \boldsymbol{F}=0$. Then for any set of finite perimeter $E \Subset \mathbb{R}^{n}$ and any $C \subset \partial^{*} E$,

$$
\int_{C} \mathscr{F} \cdot \boldsymbol{v} d \mathcal{H}^{n-1} \leqslant \frac{1}{2}\|\boldsymbol{F}\|_{\infty} \mathcal{H}^{n-1}\left(\partial^{*} E\right) .
$$


Proof. We proceed as in the proof of Corollary 3.4. For any $C \subset \partial^{*} E$, from the Gauss-Green formula (2.9) we have

$$
\mathbf{0}=\int_{\partial^{*} E} \mathscr{F} \cdot \boldsymbol{v}(x) d \mathcal{H}^{n-1}(x)=\int_{C} \mathscr{F} \cdot \boldsymbol{v}(x) d \mathcal{H}^{n-1}(x)+\int_{\partial^{*} E \backslash C} \mathscr{F} \cdot \boldsymbol{v}(x) d \mathcal{H}^{n-1}(x) .
$$

Therefore,

$$
\left|\int_{C} \mathscr{F} \cdot v(x) d \mathcal{H}^{n-1}(x)\right|=\left|\int_{\partial^{*} E \backslash C} \mathscr{F} \cdot v(x) d \mathcal{H}^{n-1}(x)\right|=\alpha(C) .
$$

Since $\mathscr{F} \cdot \boldsymbol{v} \in L^{\infty}\left(\partial^{*} E\right)$ and $\|\mathscr{F} \cdot \boldsymbol{v}\|_{\infty ; \partial^{*} E} \leqslant\|\boldsymbol{F}\|_{\infty ; \mathbb{R}^{n}}$ we obtain

$$
\alpha(C) \leqslant\|\boldsymbol{F}\|_{\infty} \mathcal{H}^{n-1}(C) \text { and } \alpha(C) \leqslant\|\boldsymbol{F}\|_{\infty} \mathcal{H}^{n-1}\left(\partial^{*} E \backslash C\right),
$$

which implies

$$
\alpha(C) \leqslant \frac{1}{2}\|\boldsymbol{F}\|_{\infty} \mathcal{H}^{n-1}\left(\partial^{*} E\right) .
$$

THEOREM 4.2 Let $\boldsymbol{F} \in L^{\infty}\left(\mathbb{R}^{n} ; \mathbb{R}^{n}\right)$ be a divergence-measure vector field satisfying div $\boldsymbol{F}=0$. Then, for any $E_{1}, E_{2}$ sets of finite perimeter with $\mathcal{L}^{n}\left(E_{2}\right)<\infty$ we have

$$
\left|\int_{\partial^{*} E_{1} \cap E_{2}^{1}} \mathscr{F} \cdot \boldsymbol{v}(x) d \mathcal{H}^{n-1}(x)\right| \leqslant \frac{1}{2}\|\boldsymbol{F}\|_{\infty} \mathcal{H}^{n-1}\left(\partial^{*} E_{2}\right)
$$

where $\mathscr{F} \cdot \boldsymbol{v}$ is the interior normal trace of $\boldsymbol{F}$ on $\partial^{*} E_{1}$.

Proof. The Gauss-Green formula (2.9) we use applies to bounded sets. To overcome this problem we, essentially, approximate $E_{2}$ by a bounded set $\hat{E}_{2}$ and then apply a limiting argument. Let $E_{3}=$ $E_{1} \cap \hat{E}_{2}$ with $\hat{E}_{2}:=\left(E_{2} \cap B\right)^{1} \cup \partial^{m}\left(E_{2} \cap B\right), B$ an open ball, and $\boldsymbol{v}_{1}, \boldsymbol{v}_{2}$ and $\boldsymbol{v}_{3}$ be the measure theoretic normal vectors corresponding to the sets $E_{1}, \hat{E}_{2}$ and $E_{3}$, respectively. We emphasize here the Šilhavý's pointwise formula for the normal trace $T_{\boldsymbol{F}}(x, \boldsymbol{v}(x))$ (see (2.7)), which depends only on the point $x$ and the normal to the set at that point, $\boldsymbol{v}(x)$. With the aid of this formula we can now use Proposition 2.9 to compute

$$
\begin{aligned}
0 & =\int_{\partial^{*} E_{3}} T_{\boldsymbol{F}}\left(x, \boldsymbol{v}_{3}(x)\right) d \mathcal{H}^{n-1}(x) \\
& =\int_{\partial^{*}\left(E_{1} \cap \hat{E}_{2}\right)} T_{\boldsymbol{F}}\left(x, \boldsymbol{v}_{3}(x)\right) d \mathcal{H}^{n-1}(x) \\
& =\int_{\partial^{*} E_{1} \cap \hat{E}_{2}^{1}} T_{\boldsymbol{F}}\left(x, \boldsymbol{v}_{1}(x)\right) d \mathcal{H}^{n-1}(x)+\int_{\partial^{*} \hat{E}_{2} \cap E_{1}^{1}} T_{\boldsymbol{F}}\left(x, \boldsymbol{v}_{2}(x)\right) d \mathcal{H}^{n-1}(x) \\
& =\int_{\partial^{*} E_{1} \cap \hat{E}_{2}^{1}} T_{\boldsymbol{F}}\left(x, \boldsymbol{v}_{1}(x)\right) d \mathcal{H}^{n-1}(x)+\int_{C} T_{\boldsymbol{F}}\left(x, \boldsymbol{v}_{2}(x)\right) d \mathcal{H}^{n-1}(x)
\end{aligned}
$$


where $C=\left(\partial^{*} \hat{E}_{2} \cap E_{1}^{1}\right) \cup S,\left(\partial^{*} \hat{E}_{2} \cap E_{1}^{1}\right) \cap S=\emptyset$ and $S \subset \partial^{*} E_{1} \cap \partial^{*} \hat{E}_{2}$ is the set where $\boldsymbol{v}_{1}(x)=\boldsymbol{v}_{2}(x)$. Therefore,

$$
\left|\int_{\partial^{*} E_{1} \cap \hat{E}_{2}^{1}} T_{\boldsymbol{F}}\left(x, \boldsymbol{v}_{1}(x)\right) d \mathcal{H}^{n-1}(x)\right|=\left|\int_{C} T_{\boldsymbol{F}}\left(x, \boldsymbol{v}_{2}(x)\right) d \mathcal{H}^{n-1}(x)\right| \leqslant \frac{1}{2}\|\boldsymbol{F}\|_{\infty} \mathcal{H}^{n-1}\left(\partial^{*} \hat{E}_{2}\right),
$$

from Lemma 4.1 applied to the set of finite perimeter $\hat{E}_{2}$. Since $\hat{E}_{2}^{1}=E_{2}^{1} \cap B$ and $\partial^{m} \hat{E}_{2}=$ $\partial^{m}\left(E_{2} \cap B\right),(4.3)$ reduces to

$$
\begin{aligned}
\left|\int_{\partial^{*} E_{1} \cap E_{2}^{1} \cap B} T_{\boldsymbol{F}}\left(x, \boldsymbol{v}_{1}(x)\right) d \mathcal{H}^{n-1}(x)\right| & \leqslant \frac{1}{2}\|\boldsymbol{F}\|_{\infty} \mathcal{H}^{n-1}\left(\partial^{*}\left(E_{2} \cap B\right)\right) \\
& \leqslant \frac{1}{2}\|\boldsymbol{F}\|_{\infty} \mathcal{H}^{n-1}\left(\partial^{*} E_{2}\right), \quad \text { by Lemma 3.5, }
\end{aligned}
$$

for any open ball $B$. We note that the condition $\mathcal{L}^{n}\left(E_{2}\right)<\infty$ is used in the previous inequality since Lemma 3.5 holds for sets of finite Lebesgue measure. We now choose concentric open balls $B_{i}, \mathbb{R}^{n}=\bigcup_{i=1}^{\infty} B_{i}$. Then, with our notation $\mathscr{F} \cdot \boldsymbol{v}(x):=T_{\boldsymbol{F}}\left(x, \boldsymbol{v}_{1}(x)\right)$, we have from (2.10) and the Dominated Convergence Theorem

$$
\begin{aligned}
\left|\int_{\partial^{*} E_{1} \cap E_{2}^{1}} \mathscr{F} \cdot \boldsymbol{v} d \mathcal{H}^{n-1}(x)\right| & =\lim _{i \rightarrow \infty}\left|\int_{\partial^{*} E_{1} \cap E_{2}^{1} \cap B_{i}} \mathscr{F} \cdot \boldsymbol{v} d \mathcal{H}^{n-1}(x)\right| \\
& \leqslant \frac{1}{2}\|\boldsymbol{F}\|_{\infty} \mathcal{H}^{n-1}\left(\partial^{*} E_{2}\right) \text { by }(4.4)
\end{aligned}
$$

and hence we conclude that

$$
\left|\int_{\partial^{*} E_{1} \cap E_{2}^{1}} \mathscr{F} \cdot \boldsymbol{v} d \mathcal{H}^{n-1}(x)\right| \leqslant \frac{1}{2}\|\boldsymbol{F}\|_{\infty} \mathcal{H}^{n-1}\left(\partial^{*} E_{2}\right) .
$$

THEOREM 4.3 Let $\boldsymbol{F} \in L^{\infty}\left(\mathbb{R}^{n} ; \mathbb{R}^{n}\right)$ be a divergence-measure vector field (i.e., div $\boldsymbol{F}=\mu \in$ $\left.\eta\left(\mathbb{R}^{n}\right)\right)$. Then, for every $E_{1}, E_{2}$ sets of finite perimeter with $\mathcal{L}^{n}\left(E_{2}\right)<\infty$ we have

$$
\left|\int_{\partial^{*} E_{1} \cap E_{2}^{1}} \mathscr{F} \cdot \boldsymbol{v}(x) d \mathcal{H}^{n-1}(x)\right| \leqslant\|\boldsymbol{F}\|_{\infty} \mathcal{H}^{n-1}\left(\partial^{*} E_{2}\right)+\|\operatorname{div} \boldsymbol{F}\|\left(E_{2}^{1}\right)
$$

where $\mathscr{F} \cdot \boldsymbol{v}$ is the interior normal trace of $\boldsymbol{F}$ on $\partial^{*} E_{1}$.

Proof. We proceed as in the previous proof and let $E_{3}=E_{1} \cap \hat{E}_{2}$ with $\hat{E}_{2}:=\left(E_{2} \cap \hat{B}\right)^{1} \cup$ $\partial^{m}\left(E_{2} \cap \hat{B}\right)$, where $\hat{B}$ is an open ball. Let $\boldsymbol{v}_{1}, \boldsymbol{v}_{2}$ and $\boldsymbol{v}_{3}$ be the measure theoretic normal vectors 
corresponding to the sets $E_{1}, \hat{E}_{2}$ and $E_{3}$, respectively. The Gauss-Green formula (2.9) yields

$$
\begin{aligned}
-\int_{E_{3}^{1}} \operatorname{div} \boldsymbol{F} & =\int_{\partial^{*} E_{3}} T_{\boldsymbol{F}}\left(x, \boldsymbol{v}_{3}(x)\right) d \mathcal{H}^{n-1}(x)=\int_{\partial^{*}\left(E_{1} \cap \hat{E}_{2}\right)} T_{\boldsymbol{F}}\left(x, \boldsymbol{v}_{3}(x)\right) d \mathcal{H}^{n-1}(x) \\
& =\int_{\partial^{*} E_{1} \cap \hat{E}_{2}^{1}} T_{\boldsymbol{F}}\left(x, \boldsymbol{v}_{1}(x)\right) d \mathcal{H}^{n-1}(x)+\int_{\partial^{*} \hat{E}_{2} \cap E_{1}^{1}} T_{\boldsymbol{F}}\left(x, \boldsymbol{v}_{2}(x)\right) d \mathcal{H}^{n-1}(x) \\
& +\int_{S} T_{\boldsymbol{F}}\left(x, \boldsymbol{v}_{2}(x)\right) d \mathcal{H}^{n-1}(x) \\
& =\int_{\partial^{*} E_{1} \cap \hat{E}_{2}^{1}} T_{\boldsymbol{F}}\left(x, \boldsymbol{v}_{1}(x)\right) d \mathcal{H}^{n-1}(x)+\int_{C} T_{\boldsymbol{F}}\left(x, \boldsymbol{v}_{2}(x)\right) d \mathcal{H}^{n-1}(x)
\end{aligned}
$$

where $C=\left(\partial^{*} \hat{E}_{2} \cap E_{1}^{1}\right) \cup S,\left(\partial^{*} \hat{E}_{2} \cap E_{1}^{1}\right) \cap S=\emptyset$ and $S \subset \partial^{*} E_{1} \cap \partial^{*} \hat{E}_{2}$ is the set where $\boldsymbol{v}_{1}(x)=\boldsymbol{v}_{2}(x)$. In this case we can not apply Lemma 4.1 since $\boldsymbol{F}$ is not divergence-free. However, we still have the following estimate

$$
\begin{aligned}
\left|\int_{\partial^{*} E_{1} \cap \hat{E}_{2}^{1}} T_{\boldsymbol{F}}\left(x, \boldsymbol{v}_{1}(x)\right) d \mathcal{H}^{n-1}(x)\right| & \leqslant\left|\int_{E_{3}^{1}} \operatorname{div} \boldsymbol{F}\right|+\left|\int_{C} T_{\boldsymbol{F}}\left(x, \boldsymbol{v}_{2}(x)\right) d \mathcal{H}^{n-1}(x)\right| \\
& \leqslant\|\operatorname{div} \boldsymbol{F}\|\left(E_{3}^{1}\right)+\|\boldsymbol{F}\|_{\infty} \mathcal{H}^{n-1}(C) \\
& \leqslant\|\operatorname{div} \boldsymbol{F}\|\left(E_{3}^{1}\right)+\|\boldsymbol{F}\|_{\infty} \mathcal{H}^{n-1}\left(\partial^{*} \hat{E}_{2}\right) .
\end{aligned}
$$

We now proceed to show that

$$
E_{3}^{1} \subset E_{2}^{1} \text {. }
$$

Indeed, let $x \in E_{3}^{1}$. This means that

$$
\lim _{r \rightarrow 0} \frac{\mathcal{L}^{n}\left(E_{3} \cap B(x, r)\right)}{\mathcal{L}^{n}(B(x, r))}=1,
$$

and since $\partial^{m}\left(E_{2} \cap \hat{B}\right)$ has $\mathcal{L}^{n}$-measure zero, this is equivalent to

$$
\lim _{r \rightarrow 0} \frac{\mathcal{L}^{n}\left(E_{1} \cap E_{2}^{1} \cap \hat{B} \cap B(x, r)\right)}{\mathcal{L}^{n}(B(x, r))}=1 .
$$

Notice that (4.7) implies that $x \in \hat{B}$. Thus, for $r$ small enough we have $B(x, r) \subset \hat{B}$ and (4.7) becomes

$$
\lim _{r \rightarrow 0} \frac{\mathcal{L}^{n}\left(E_{1} \cap E_{2}^{1} \cap B(x, r)\right)}{\mathcal{L}^{n}(B(x, r))}=1,
$$

and since

$$
\frac{\mathcal{L}^{n}\left(E_{1} \cap E_{2}^{1} \cap B(x, r)\right)}{\mathcal{L}^{n}(B(x, r))} \leqslant \frac{\mathcal{L}^{n}\left(E_{2} \cap B(x, r)\right)}{\mathcal{L}^{n}(B(x, r))} \leqslant 1,
$$

if follows from (4.8) that

$$
\lim _{r \rightarrow 0} \frac{\mathcal{L}^{n}\left(E_{2} \cap B(x, r)\right)}{\mathcal{L}^{n}(B(x, r))}=1,
$$


which means that $x \in E_{2}^{1}$. Furthermore, since $\hat{E}_{2}^{1}=E_{2}^{1} \cap \hat{B}$ and $\partial^{m} \hat{E}_{2}=\partial^{m}\left(E_{2} \cap \hat{B}\right)$, (4.6) becomes

$$
\left|\int_{\partial^{*} E_{1} \cap E_{2}^{1} \cap \hat{B}} T_{\boldsymbol{F}}\left(x, \boldsymbol{v}_{1}(x)\right) d \mathcal{H}^{n-1}(x)\right| \leqslant\|\operatorname{div} \boldsymbol{F}\|\left(E_{2}^{1}\right)+\|\boldsymbol{F}\|_{\infty} \mathcal{H}^{n-1}\left(\partial^{*} E_{2}\right)
$$

Since (4.10) is true for every open ball $\hat{B}$, we proceed as in Theorem 4.2 to conclude the desired result.

\section{Limits of sets with perimeter growing to $\infty$}

It is well known that when a sequence of sets $E_{i}$, with $\chi_{E_{i}} \in B V\left(\mathbb{R}^{n}\right)$, converges in $L^{1}$ to a set $E_{0}$, then $E_{0}$ satisfies

$$
\mathcal{H}^{n-1}\left(\partial^{*} E_{0}\right) \leqslant \liminf _{i \rightarrow \infty} \mathcal{H}^{n-1}\left(\partial^{*} E_{i}\right) .
$$

In this section, we consider two degenerate cases. The first when the perimeter grows indefinitely, and the second when the Lebesgue measure shrinks to zero. Specifically, the limit is studied by means of occupational measures. The results from this section will be applied to shape optimization in a forthcoming paper.

For every set of finite perimeter we define a corresponding probability measure in the following manner.

Definition 5.1. We define the occupational measure $\mu \in P\left(\mathbb{R}^{n} \times \mathbb{S}^{n-1}\right)$ corresponding to a set of finite perimeter $E$ by

$$
\mu(U \times V)=\frac{1}{\mathcal{H}^{n-1}\left(\partial^{*} E\right)} \mathfrak{H}^{n-1}\left(x \in \partial^{*} E \mid(x, v(x)) \in U \times V\right),
$$

for every Borel sets $U \subset \mathbb{R}^{n}$ and $V \subset \mathbb{S}^{n-1}$.

A useful property of occupational measures is that, for every continuous function $g \in$ $C\left(\mathbb{R}^{n} \times \mathbb{S}^{n-1}\right)$ we have that

$$
\frac{1}{\mathcal{H}^{n-1}\left(\partial^{*} E\right)} \int_{\partial^{*} E} g(x, v(x)) d \mathcal{H}^{n-1}(x)=\int_{\mathbb{R}^{n} \times \mathbb{S}^{n-1}} g(x, v) d \mu(x, v) .
$$

We endow the set of probability measures $P\left(\mathbb{R}^{n} \times \mathbb{S}^{n-1}\right)$ with the weak topology, namely, a sequence of measures $\mu_{1}, \mu_{2}, \cdots \in P\left(\mathbb{R}^{n} \times \mathbb{S}^{n-1}\right)$ converges weakly to a measure $\mu_{0} \in$ $P\left(\mathbb{R}^{n} \times \mathbb{S}^{n-1}\right)$ if for every bounded continuous function $g \in C\left(\mathbb{R}^{n} \times \mathbb{S}^{n-1}\right)$,

$$
\lim _{i \rightarrow \infty} \int_{\mathbb{R}^{n} \times \mathbb{S}^{n-1}} g(x, v) d \mu_{i}(x, v)=\int_{\mathbb{R}^{n} \times \mathbb{S}^{n-1}} g(x, v) d \mu_{0}(x, v) .
$$

Furthermore, if the sequence $\mu_{1}, \mu_{2}, \cdots \in P\left(\mathbb{R}^{n} \times \mathbb{S}^{n-1}\right)$ converges weakly to $\mu_{0}$, and $f(x, v)$ is a bounded Borel function with compact support such that the set of its discontinuity points is $\mu_{0}$-negligible, then (see Ambrosio-Fusco-Pallara [1, Proposition 1.62, pages 27-28].)

$$
\lim _{i \rightarrow \infty} \int_{\mathbb{R}^{n} \times \mathbb{S}^{n-1}} f(x, v) d \mu_{i}(x, v)=\int_{\mathbb{R}^{n} \times \mathbb{S}^{n-1}} f(x, v) d \mu_{0}(x, v) .
$$


The space $P\left(K \times \mathbb{S}^{d-1}\right)$ is compact in the weak topology, whenever $K \subset \mathbb{R}^{n}$ is compact (see, Billingsley [4, page 72]).

Another tool we need for the next theorem is disintegration of measures. Given a probability measure $\mu \in P\left(\mathbb{R}^{n} \times \mathbb{S}^{n-1}\right)$, we denote its disintegration by $\mu=p \circledast \mu^{x}$; the marginal measure is $p \in P\left(\mathbb{R}^{n}\right)$, which is the push forward of the projection map $\pi: \mathbb{R}^{n} \times \mathbb{S}^{n-1} \rightarrow \mathbb{R}^{n}$; that is $p=\pi_{\#} \mu$, and $p(A)=\mu\left(A \times \mathbb{S}^{n-1}\right)$ for every Borel set $A \subset \mathbb{R}^{n}$. The measure-valued function $\mu^{x} \in P\left(\mathbb{S}^{n-1}\right)$ is the disintegration with respect to $p$, for $p$-almost every $x$. With this notation, for every Borel sets $C \subset \mathbb{R}^{n}$ and $D \subset \mathbb{S}^{n-1}$, we have that $\mu(C \times D)=\int_{C} \mu^{x}(D) d p(x)$.

Note that when $\mu$ is the occupational measure of a set of finite perimeter, then the disintegration is a Dirac measure $p$-almost everywhere.

THEOREM 5.2 Let $E_{1}, E_{2}, \cdots \subset \mathbb{R}^{n}$ be sets of finite perimeter, with perimeter growing to infinity, namely, $\lim _{i \rightarrow \infty} \mathcal{H}^{n-1}\left(\partial^{*} E_{i}\right)=\infty$. If the corresponding sequence of occupational measures $\mu_{1}, \mu_{2}, \ldots$ converges weakly to $\mu_{0} \in P\left(\mathbb{R}^{n} \times \mathbb{S}^{n-1}\right)$ then

$$
h(x)=\int_{\mathbb{S}^{n-1}} v d \mu_{0}^{x}(v)=0,
$$

for $p_{0}$-almost every $x$, where $\mu_{0}=p_{0} \circledast \mu_{0}^{x}$ is the disintegration of $\mu_{0}$ with respect to its projection, $p_{0}$.

Proof. Let us fix $x$ in the support of $p_{0}$. By the definition of the occupational measure and Theorem 3.2 we obtain, for every $i$,

$$
\left|\int_{B(x, r) \times \mathbb{S}^{n-1}} v d \mu_{i}(y, v)\right|=\left|\frac{1}{\mathcal{H}^{n-1}\left(\partial^{*} E_{i}\right)} \int_{\partial^{*} E_{i} \cap B(x, r)} v(y) d \mathcal{H}^{n-1}(y)\right| \leqslant \frac{\mathcal{H}^{n-1}(\partial B(x, r))}{2 \mathcal{H}^{n-1}\left(\partial^{*} E_{i}\right)} .
$$

The weak convergence implies that

$$
\left|\int_{B(x, r) \times \mathbb{S}^{n-1}} v d \mu_{0}(y, v)\right|=\lim _{i \rightarrow \infty}\left|\int_{B(x, r) \times \mathbb{S}^{n-1}} v d \mu_{i}(y, v)\right| \leqslant \lim _{i \rightarrow \infty} \frac{\mathcal{H}^{n-1}(\partial B(x, r))}{2 \mathcal{H}^{n-1}\left(\partial^{*} E_{i}\right)}=0,
$$

for almost every $r>0$, for which $\mu_{0}\left(\partial B(x, r) \times \mathbb{S}^{d-1}\right)=0$. With the disintegration notation one obtains

$$
\left|\int_{B(x, r) \times \mathbb{S}^{n-1}} v d \mu_{0}(y, v)\right|=\left|\int_{B(x, r)}\left(\int_{\mathbb{S}^{n-1}} v d \mu_{0}^{y}(v)\right) d p_{0}(y)\right|=\int_{B(x, r)} h(y) d p_{0}(y)=0,
$$

for almost every $r>0$.

If the measure $p_{0}$ was the Lebesgue measure, by the Lebesgue differentiation theorem $h(x)=0$ almost everywhere. The Lebesgue-Besicovitch differentiation theorem extends this result to Radon measures (see Evans-Gariepy [11, page 43]).

THEOREM 5.3 Let $E_{1}, E_{2}, \cdots \subset \mathbb{R}^{n}$ be sets of finite perimeter. If $\lim _{i \rightarrow \infty} \mathcal{L}^{n}\left(E_{i}\right)=0$ and the corresponding sequence of occupational measures $\mu_{1}, \mu_{2}, \ldots$ converges weakly to $\mu_{0} \in$ $P\left(\mathbb{R}^{n} \times \mathbb{S}^{n-1}\right)$ then

$$
h(x)=\int_{\mathbb{S}^{n-1}} v d \mu_{0}^{x}(v)=0
$$

for $p_{0}$-almost every $x$, where $\mu_{0}=p_{0} \circledast \mu_{0}^{x}$ is the disintegration of $\mu_{0}$ with respect to its projection, $p_{0}$. 
Proof. Let us fix $x$ in the support of $p_{0}$. From Lemma 2.10 it follows that $\mathcal{H}^{n-1}\left(\partial^{*} E_{i} \cap \partial B(x, r)\right)=$ 0 , for almost every $r>0$ and every $i=1,2, \ldots$. Hence, Lemma 3.3 and Proposition 2.9 (1) applied to $E_{i} \cap B(x, r)$ imply, for almost every $r>0$ and every $i=1,2, \ldots$,

$$
\begin{aligned}
\mathbf{0} & =\int_{\partial^{*}\left(E_{i} \cap B(x, r)\right)} \boldsymbol{v}(y) d \mathcal{H}^{n-1}(y) \\
& =\int_{\partial^{*} E_{i} \cap B(x, r)} \boldsymbol{v}(y) d \mathcal{H}^{n-1}(y)+\int_{\partial B(x, r) \cap E_{i}} \boldsymbol{v}(y) d \mathcal{H}^{n-1}(y),
\end{aligned}
$$

and therefore,

$$
\begin{aligned}
\left|\int_{B(x, r) \times \mathbb{S}^{n-1}} v d \mu_{i}(y, v)\right| & =\frac{1}{\mathcal{H}^{n-1}\left(\partial^{*} E_{i}\right)}\left|\int_{\partial^{*} E_{i} \cap B(x, r)} v(y) d \mathcal{H}^{n-1}(y)\right| \\
& =\frac{1}{\mathcal{H}^{n-1}\left(\partial^{*} E_{i}\right)}\left|\int_{E_{i} \cap \partial B(x, r)} v(y) d \mathcal{H}^{n-1}(y)\right| \\
& \leqslant \frac{\mathcal{H}^{n-1}\left(\partial B(x, r) \cap E_{i}\right)}{\mathcal{H}^{n-1}\left(\partial^{*} E_{i}\right)} .
\end{aligned}
$$

We integrate both sides of the previous inequality with respect to $\mathcal{L}(r)$ and use the coarea formula to obtain

$$
\begin{aligned}
\int_{0}^{1}\left|\int_{B(x, r) \times \mathbb{S}^{n-1}} v d \mu_{i}(y, v)\right| d \mathcal{L}(r) & \leqslant \frac{1}{\mathcal{H}^{n-1}\left(\partial^{*} E_{i}\right)} \int_{0}^{1} \mathcal{H}^{n-1}\left(\partial B(x, r) \cap E_{i}\right) d \mathcal{L}(r) \\
& \leqslant \frac{1}{\mathcal{H}^{n-1}\left(\partial^{*} E_{i}\right)} \int_{0}^{\infty} \mathcal{H}^{n-1}\left(\partial B(x, r) \cap E_{i}\right) d \mathcal{L}(r) \\
& =\frac{\mathcal{L}^{n}\left(E_{i}\right)}{\mathcal{H}^{n-1}\left(\partial^{*} E_{i}\right)} \\
& =\frac{\mathcal{L}^{n}\left(E_{i}\right)^{\frac{1}{n}} \mathcal{L}^{n}\left(E_{i}\right)^{\frac{n-1}{n}}}{\mathcal{H}^{n-1}\left(\partial^{*} E_{i}\right)} .
\end{aligned}
$$

By the isoparametric inequality there exists a constant $C_{1}$, depending only on the dimension $n$, such that $\mathcal{L}^{n}(E)^{(n-1) / n} \leqslant C_{1} \mathcal{H}^{n-1}\left(\partial^{*} E\right)$ for every set of finite perimeter $E$. Hence, (5.1) is bounded by $C_{1}\left(\mathscr{L}^{n}\left(E_{i}\right)\right)^{1 / n}$. The weak convergence and Fatou's lemma imply that

$$
\begin{aligned}
\int_{0}^{1}\left|\int_{B(x, r) \times \mathbb{S}^{n-1}} v d \mu_{0}(y, v)\right| d \mathcal{L}(r) & =\int_{0}^{1} \lim _{i \rightarrow \infty}\left|\int_{B(x, r) \times \mathbb{S}^{n-1}} v d \mu_{i}(y, v)\right| d \mathcal{L}(r) \\
& \leqslant \liminf _{i \rightarrow \infty} \int_{0}^{1}\left|\int_{B(x, r) \times \mathbb{S}^{n-1}} v d \mu_{i}(y, v)\right| d \mathcal{L}(r) \\
& \leqslant \liminf _{i \rightarrow \infty} C_{1}\left(\mathcal{L}^{n}\left(E_{i}\right)\right)^{1 / n}=0 .
\end{aligned}
$$

Applying a disintegration argument similar to Theorem 5.2 we complete the proof.

Remark 5.4. Theorem 5.3 holds when $\lim _{i \rightarrow \infty} \min \left(\mathcal{L}^{n}\left(E_{i}\right), \mathcal{L}^{n}\left(\mathbb{R}^{n}-E_{i}^{1}\right)\right)=0$, since replacing $E_{i}$ by $\mathbb{R}^{n}-E_{i}^{1}$, when needed, only changes the sign of the measurable normal vector, and as we take the absolute value of all our integrals, it will not affect our final result. 


\section{REFERENCES}

1. Ambrosio, L., Fusco, N. \& Pallara, D., Functions of Bounded Variation and Free Discontinuity Problems. Oxford Mathematical Monographs. The Clarendon Press, Oxford University Press: New York, 2000. Zb10957. 49001 MR1857292

2. Artstein, Z. \& BRight, I., Periodic optimization suffices for infinite horizon planar optimal control. SIAM J. Control Optim. 48 (2010), 4963-4986. Zbl1208.49006 MR2735512

3. BRIght, I. \& LEE, J. M., A note on flux integrals over smooth regular domains. Pacific Journal of Mathematics 272 (2014), 305-322. Zbl06406047 MR3284889

4. Billingsley, P., Convergence of probability measures. Wiley-Interscience, 2009.

5. Caffarelli, L. A. \& De la Llave, R., Planelike minimizers in periodic media. Comm. Pure Appl. Math. 54 (2001), 1403-1441. Zbl1036.49040 MR1852978

6. Chambolle, A., Goldman, M. \& Novaga, M., Fine properties of the subdifferential for a class of one-homogeneous functionals. Adv. Calc. Var. 8 (2015), 31-42. Zbl06387043 MR3297723

7. Chen, G.-Q. \& FRID, H., Divergence-measure fields and hyperbolic conservation laws. Arch. Ration. Mech. Anal. 147 (1999), 89-118. Zbl0942.35111 MR1702637

8. Chen, G.-Q. \& Torres, M., Divergence-measure fields, sets of finite perimeter, and conservation laws. Arch. Rational Mech. Anal. 175 (2005), 245-267. Zbl1073.35156 MR2118477

9. Chen,G.-Q., Torres, M. \& Ziemer, W.P., Gauss-Green Theorem for weakly differentiable vector fields, sets of finite perimeter, and balance laws. Communications on Pure and Applied Mathematics 62 (2009), 242-304. Zb11158.35062 MR2468610

10. Dafermos, C. M., Hyperbolic Conservation Laws in Continuum Physics, 2nd Ed., Springer Verlag: Berlin, 2005. Zbl1078.35001 MR2169977

11. Evans, C. \& GARIEPY, D., Measure Theory and Fine Properties of Functions. CRC Press, Boca Raton, FL, 1992. Zb10804.28001 MR1158660

12. Federer, H., Geometric Measure Theory. Springer Verlag, New York Inc, 1969. Zb10176.00801 MR0257325

13. Finlay, L., Gaitsgory, V. \& Lebedev, I., Duality in linear programming problems related to deterministic long run average problems of optimal control. SIAM J. Control Optim. 47 (2008), 16671700. Zb11167. 49032 MR2421325

14. FRID, H., Remarks on the theory of the divergence-measure fields. Quartely of Applied Mathematics $\mathbf{7 0}$ (2012), 579-596. Zb106081551 MR2986135

15. GAITSGORY, V. \& QuinCAMPOIX, M., Linear programming approach to deterministic infinite horizon optimal control problems with discounting. SIAM J. Control Optim. 48 (2009), 2480-2512. Zbl1201. 49040 MR2556353

16. ŠILhaVÝ, M., The mechanics and thermodynamics of continuous media. Springer Verlag, 1997. Zb10870.73004 MR1423807

17. ŠILhavÝ, M., Divergence measure fields and Cauchy's stress theorem. Rend. Sem. Mat. Univ. Padova 113 (2005), 15-45. Zbl1167.74317 MR2168979

18. ZIEMER, W. P., Weakly Differentiable Functions, Graduate Texts in Mathematics. Springer Verlag: New York, 120, 1989. Zbl0692.46022 MR1014685 